\title{
Design Methodology of a Passive Vibration Isolation System for an Optical System With Sensitive Line-of-Sight
}

\author{
Keqi QI ${ }^{1,2}$, Lei DAI ${ }^{1}$, Shaoxin $\mathrm{WANG}^{1}$, Yongming $\mathrm{YANG}^{3}$, \\ Yalin $\mathrm{DING}^{3}$, Chao FANG ${ }^{1}$, and Chao LIN $^{1 *}$ \\ ${ }^{1}$ State key Laboratory of Applied Optics, Changchun Institute of Optics, Fine Mechanics and Physics, Chinese Academy \\ of Science, Changchun 130033, China \\ ${ }^{2}$ University of Chinese Academy of Sciences, Beijing 100049, China \\ ${ }^{3}$ Key Laboratory of Airborne Optical Imaging and Measurement, Changchun Institute of Optics, Fine Mechanics and \\ Physics, Chinese Academy of Science, Changchun 130033, China \\ *Corresponding author: Chao LIN_ E-mail: address: linchaoluck@163.com
}

\begin{abstract}
The performance of an optical system with sensitive line-of-sight (LOS) is influenced by rotational vibration. In view of this, a design methodology is proposed for a passive vibration isolation system in an optical system with sensitive LOS. Rotational vibration is attributed to two sources: transmitted from the mounting base and generated by modal coupling. Therefore, the elimination of the rotational vibration caused by coupling becomes an important part of the design of the isolation system. Additionally, the decoupling conditions of the system can be obtained. When the system is totally decoupled, the vibration on each degree of freedom (DOF) can be analyzed independently. Therefore, the stiffness and damping coefficient on each DOF could be obtained by limiting the vibration transmissibility, in accordance to actual requirements. The design of a vibration isolation system must be restricted by the size and shape of the payload and the installation space, and the layout constrains are thus also discussed.
\end{abstract}

Keywords: Optical system with sensitive LOS; passive vibration isolation system; decoupling conditions; transmissibility

Citation: Keqi QI, Lei DAI, Shaoxin WANG, Yongming YANG, Yalin DING, Chao FANG, et al., "Design Methodology of a Passive Vibration Isolation System for an Optical System With Sensitive Line-of-Sight," Photonic Sensors, 2021, 11(4): $435-447$.

\section{Introduction}

In most optical systems, vibration causes the undesired motion of its components and leads to performance loss [1-7]. It can downgrade the precision of sensitive optical telescopes, cause the misalignment of laser communication devices, and induce blurriness in the images of airborne cameras [8-13]. Thus, the suppression and isolation of vibration are essential concerns for high performance optical systems.

Vibration can be classified in two categories: translational vibration and rotational vibration [14, 15]. Some optical systems such as astronomical telescopes, laser communication devices, and long focal length airborne cameras have very sensitive line-of-sight (LOS) and require very high pointing accuracies [16-22]. For example, the National

Received: 27 August 2020 / Revised: 24 November 2020

(C) The Author(s) 2021. This article is published with open access at Springerlink.com

DOI: $10.1007 / \mathrm{s} 13320-021-0610-0$

Article type: Regular 
Aeronautics and Space Administration (NASA) Hubble Space Telescope (HST) requires a telescope pointing accuracy of $0.01 \operatorname{arcsec}$ [9], while the pointing accuracy of the deep space optical communication (DSOC) antenna is just a few milliradians [23]. Figure 1(a) shows the image shift of a long focal length airborne camera caused by translational vibration, and the image shift can be obtained by $\delta=d^{\prime}-d=D(f / H)$, where $D$ is the amplitude of translational vibration, $f$ is the focal length of the camera, $H$ is the flight altitude of the aircraft, $d$ is the distance between the image of the target on the CCD and the center of the CCD before the vibration occurs, and $d^{\prime}$ is the distance between the image of the target on the CCD and the center of the CCD after the vibration occurs. Figure 1(b) shows the image shift of the same airborne camera caused by the rotational vibration, and the image shift can be obtained by $\delta^{\prime}=f \cdot \sin (\alpha)$, where $\alpha$ is the amplitude of rotational vibration. When $H=10 \mathrm{~km}$, $f=880 \mathrm{~mm}, D=10 \mathrm{~mm}$, and $\alpha=30 \mathrm{arcsec}$, the image shifts are $\delta=0.88 \mu \mathrm{m}$ and $\delta^{\prime}=128 \mu \mathrm{m}$, respectively. For this optical system type, the performance loss caused by the rotational vibration is far more than that caused by the translational vibration. Therefore, the primary task of designing the vibration isolation systems of optical systems with the sensitive LOS is to eliminate the rotational vibration. In fact, for optical systems without the internal vibration, the rotational vibration comes from two sources: transmitted from the mounting base and generated by modal coupling, as shown in Fig. 2. Generally, the rotational vibration transmitted from the mounting base can be predicted by analyzing the working environment and suppressed or isolated by a well-designed vibration isolation system. However, in most instances, the rotational vibration generated by modal coupling is unexpected, which is difficult to predict and eliminated. Therefore, designing an uncoupled vibration isolation system is very important for an optical system with the sensitive LOS.

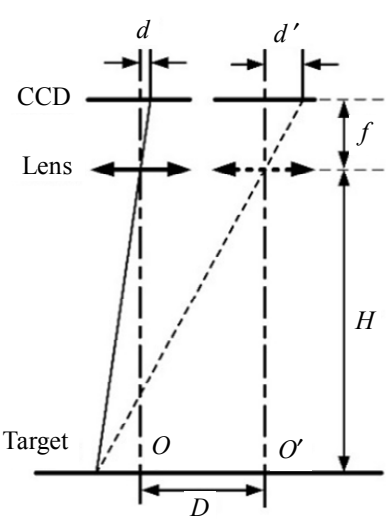

(a)

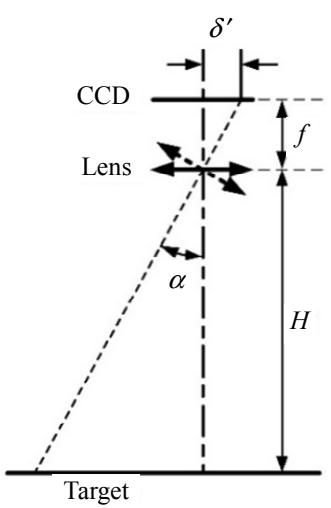

(b)
Fig. 1 Image shift of a long focal length airborne camera caused by (a) translational vibration and (b) rotational vibration.

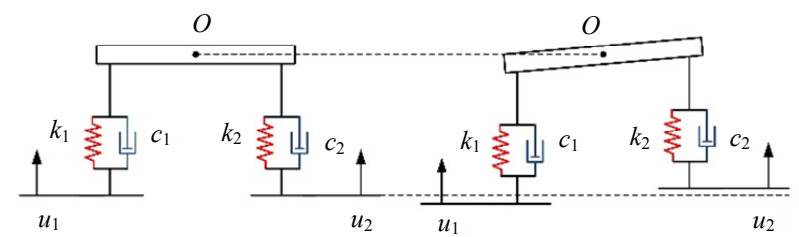

(a)

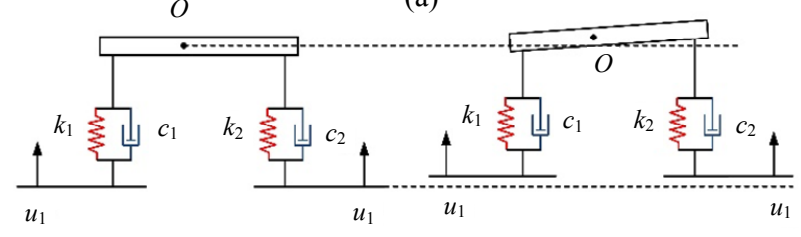

(b)

Fig. 2 Sources of the rotational vibration: (a) transmitted from the mounting base and (b) generated by modal coupling.

According to different application purposes, the vibration isolation system can be divided into two categories: one is used as the support of the vibration source to prevent the generation of vibration [24], and the other is used as the support of the sensitive payload to prevent the vibration [25]. Furthermore, depending on these means, the vibration isolation system can also be classified into four different types: passive, active, active-passive hybrid, and semiactive [26]. Davis et al. [27] designed a passive viscous damping strut (D-strut) with a very low fundamental frequency to isolate disturbances from a reaction wheel assembly (RWA). Cobb et al. [28] designed a vibration isolation and suppression system (VISS) to isolate a precision payload from spacecraft borne disturbances using passive isolation in combination with voice coil 
actuators. This research study mainly focuses on the passive vibration isolation for the prevention of the transmission of vibration to the optical payloads.

To obtain an effective, low-cost, and reliable solution, the vibration isolation should be considered from the beginning of the conceptual design of the sensitive optical system. O'Toole [29] discussed the design of a passive isolation system for a high-altitude and long-range oblique reconnaissance camera, and introduced some key factors for passive isolation designs, including the selection and layout of isolators. Hyde [30] proposed a conceptual design methodology for the vibration isolation, including the conceptual design process, performance target allocation, and design tradeoffs. However, none of them provided detailed theoretical analysis and derivation in all degrees of freedom (DOFs).

This study proposes a design methodology of a passive vibration isolation system for an optical system with the sensitive LOS. The remaining parts of this study are organized as follows. The dynamic model of the passive vibration isolation system is established in Section 2. The modal coupling characteristics are analyzed in Section 3. Section 4 introduces the method of selecting the suitable vibration transmissibility. In Section 5, the layout constrains are discussed. Concluding remarks are summarized in Section 6.

\section{Dynamic model}

An optical payload supported by $n$ isolators is shown in Fig. 3. $m$ is the mass of the payload, and $O$ is the center of mass and the origin of a Cartesian coordinate system. In this case, the coordinate axes are selected to coincide with the principal inertial axes of the payload. The simplified model of the vibration isolation system of the optical payload is shown in Fig. 4, where $k_{x 1}, k_{x 2}, k_{x i}$, and $k_{x n}$, are the stiffnesses of Isolators $1,2, i$, and $n$, along the $X$-axis, respectively; $k_{y 1}, k_{y 2}, k_{y i}$, and $k_{y n}$, are the stiffnesses of Isolators $1,2, i$, and $n$, along the $Y$-axis, respectively; $k_{z 1}, k_{z 2}, k_{z i}$, and $k_{\mathrm{zn}}$, are the stiffnesses of
Isolators $1,2, i$, and $n$, along the $Z$-axis, respectively; $c_{x 1}, c_{x 2}, c_{x i}$, and $c_{x n}$, are the damping coefficients of Isolators $1,2, i$, and $n$, along the $X$-axis, respectively; $c_{y 1}, c_{y 2}, c_{y i}$, and $c_{y n}$, are the damping coefficients of Isolators $1,2, i$, and $n$, along the $Y$-axis, respectively; and $c_{z 1}, c_{z 2}, c_{z i}$, and $c_{z n}$, are the damping coefficients of Isolators $1,2, i$, and $n$, along the $Z$-axis, respectively.

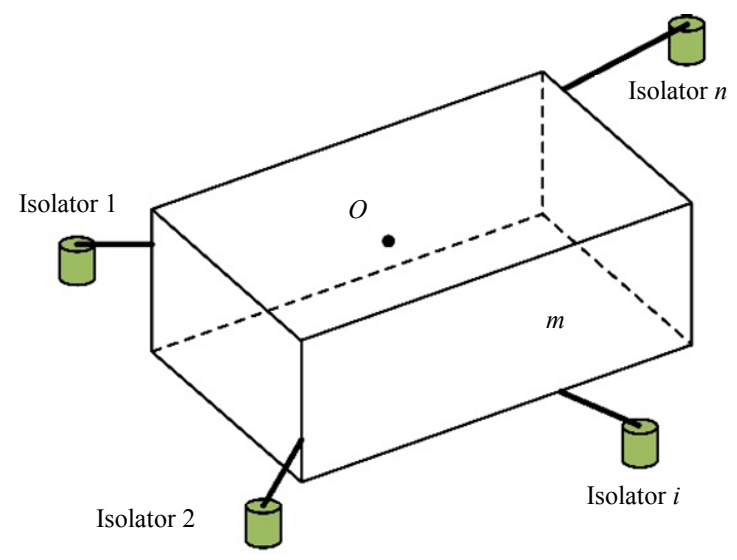

Fig. 3 Schematic diagram of an optical payload supported by $n$ isolators (only four isolators are displayed).

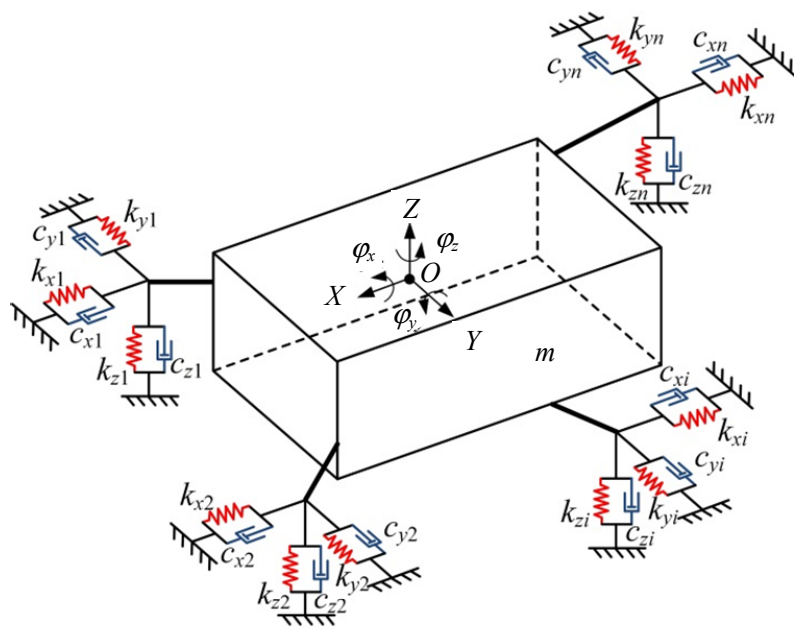

Fig. 4 Simplified model of the vibration isolation system of the optical payload.

Based on Newton's second law of motion, the dynamic equations of the vibration isolation system can be expressed in accordance to the following equations: 


$$
\begin{aligned}
& {\left[\begin{array}{ccccccc}
m & & & & \\
& m & & & & \\
& m & & & \\
& & I_{x x} & & \\
& & & I_{y y} & \\
& & & \\
& & & I_{z z}
\end{array}\right]\left[\begin{array}{c}
\ddot{x} \\
\ddot{y} \\
\ddot{z} \\
\ddot{\varphi}_{x} \\
\ddot{\varphi}_{y} \\
\ddot{\varphi}_{z}
\end{array}\right]+\left[\begin{array}{llllll}
C_{x} & & & & Q_{x z} & Q_{x y} \\
& C_{y} & & Q_{y z} & & Q_{y x} \\
& & C_{z} & Q_{z y} & Q_{z x} & \\
& Q_{y z} & Q_{z y} & C_{x x} & C_{x y} & C_{x z} \\
Q_{x z} & & Q_{z x} & C_{y x} & C_{y y} & C_{y z} \\
Q_{x y} & Q_{y x} & & C_{z x} & C_{z y} & C_{z z}
\end{array}\right]\left[\begin{array}{c}
\dot{x} \\
\dot{y} \\
\dot{z} \\
\dot{\varphi}_{x} \\
\dot{\varphi}_{y} \\
\dot{\varphi}_{z}
\end{array}\right]+} \\
& {\left[\begin{array}{cccccc}
K_{x} & & & & R_{x z} & R_{x y} \\
& K_{y} & & R_{y z} & & R_{y x} \\
& & K_{z} & R_{z y} & R_{z x} & \\
& R_{y z} & R_{z y} & K_{x x} & K_{x y} & K_{x z} \\
R_{x z} & & R_{z x} & K_{y x} & K_{y y} & K_{y z} \\
R_{x y} & R_{y x} & & K_{z x} & K_{z y} & K_{z z}
\end{array}\right]\left[\begin{array}{c}
x \\
y \\
z \\
\varphi_{x} \\
\varphi_{y} \\
\varphi_{z}
\end{array}\right]=\left[\begin{array}{c}
F_{x} \\
F_{y} \\
F_{z} \\
M_{x} \\
M_{y} \\
M_{z}
\end{array}\right]}
\end{aligned}
$$

where $I_{x x}, I_{y y}$, and $I_{z z}$, are the moments of inertia of the payload with respect to the $X$-, $Y$-, and $Z$-axes, respectively; $F_{x}, F_{y}$, and $F_{z}$, are the excitation forces acting on the center of mass along the $X-, Y_{-}$, and $Z$-axes, respectively; $M_{x}, M_{y}$, and $M_{z}$, are the excitation moments acting on the center of mass with respect to the $X$-, $Y$-, and $Z$-axes, respectively. Moreover, the parameters of the stiffness and damping matrices can be derived from the following equations:

$$
\begin{aligned}
& K_{x}=\sum_{1}^{n} k_{x i}, K_{y}=\sum_{1}^{n} k_{y i}, K_{z}=\sum_{1}^{n} k_{z i}, K_{x x}=\sum_{1}^{n}\left(k_{y i} l_{z i}^{2}+k_{z i} l_{y i}^{2}\right), K_{y y}=\sum_{1}^{n}\left(k_{x i} l_{z i}^{2}+k_{z i} l_{x i}^{2}\right), K_{z z}=\sum_{1}^{n}\left(k_{x i} l_{y i}^{2}+k_{y i} l_{x i}^{2}\right) \\
& C_{x}=\sum_{1}^{n} c_{x i}, C_{y}=\sum_{1}^{n} c_{y i}, C_{z}=\sum_{1}^{n} c_{z i}, C_{x x}=\sum_{1}^{n}\left(c_{y i} l_{z i}^{2}+c_{z i} l_{y i}^{2}\right), C_{y y}=\sum_{1}^{n}\left(c_{x i} l_{z i}^{2}+c_{z i} l_{x i}^{2}\right), C_{z z}=\sum_{1}^{n}\left(c_{x i} l_{y i}^{2}+c_{y i} l_{x i}^{2}\right) \\
& K_{x y}=K_{y x}=\sum_{1}^{n} k_{z i} l_{x i} l_{y i}, K_{x z}=K_{z x}=\sum_{1}^{n} k_{y i} l_{x i} l_{z i}, K_{y z}=K_{z y}=\sum_{1}^{n} k_{x i} l_{y i} l_{z i} \\
& C_{x y}=C_{y x}=\sum_{1}^{n} c_{z i} l_{x i} l_{y i}, C_{x z}=C_{z x}=\sum_{1}^{n} c_{y i} l_{x i} l_{z i}, C_{y z}=C_{z y}=\sum_{1}^{n} c_{x i} l_{y i} l_{z i} \\
& R_{x y}=\sum_{1}^{n} k_{x i} l_{y i}, R_{y x}=\sum_{1}^{n} k_{y i} l_{x i}, R_{y z}=\sum_{1}^{n} k_{y i} l_{z i} R_{z y}=\sum_{1}^{n} k_{z i} l_{y i}, R_{z x}=\sum_{1}^{n} k_{z i} l_{x i}, R_{x z}=\sum_{1}^{n} k_{x i} l_{z i} \\
& Q_{x y}=\sum_{1}^{n} c_{x i} l_{y i}, Q_{y x}=\sum_{1}^{n} c_{y i} l_{x i}, Q_{y z}=\sum_{1}^{n} c_{y i} l_{z i}, Q_{z y}=\sum_{1}^{n} c_{z i} l_{y i}, Q_{z x}=\sum_{1}^{n} c_{z i} l_{x i}, Q_{x z}=\sum_{1}^{n} c_{x i} l_{z i} \\
& F_{x}=\sum_{1}^{n} F_{x i}, F_{y}=\sum_{1}^{n} F_{y i}, F_{z}=\sum_{1}^{n} F_{z i}, M_{x}=\sum_{1}^{n} M_{x i}, M_{y}=\sum_{1}^{n} M_{y i}, M_{z}=\sum_{1}^{n} M_{z i}
\end{aligned}
$$

where $l_{x i}, l_{y i}$, and $l_{z i}$ are the coordinates of Isolator $i$ on the $X$-, $Y$ - and $Z$-axes, respectively.

Equation(1) can be expressed as

$$
\mathbf{M} \ddot{\mathbf{X}}+\mathbf{C} \dot{\mathbf{X}}+\mathbf{K X}=\mathbf{F}
$$

where $\mathbf{M}, \mathbf{C}$, and $\mathbf{K}$ represent the mass, damping, and the stiffness matrices of (1), respectively, and F represents the excitation load vector of (1).
Suppose that the initial position and the initial velocities of the vibration isolation system are zero. After the application of the Laplace transformation, (3) can be written as

$$
\left[\mathbf{M} s^{2}+\mathbf{C} s+\mathbf{K}\right] \mathbf{X}(s)=\boldsymbol{\Phi}(s)
$$

where $\left[\mathbf{M} s^{2}+\mathbf{C} s+\mathbf{K}\right]$ is the impedance matrix of the system, represented by $\mathbf{Z}(s)$. 


\section{Decoupling condition analysis}

From (1), it can be inferred that the impedance matrix is nonsingular. Therefore, this matrix is reversible. Correspondingly, we can obtain that

$$
\mathbf{X}(s)=\left[\mathbf{M} s^{2}+\mathbf{C} s+\mathbf{K}\right]^{-1} \mathbf{\Phi}(s)
$$

where $\left[\mathbf{M} s^{2}+\mathbf{C} s+\mathbf{K}\right]^{-1}$ is the admittance matrix of the system.

If we only consider the translational DOF of the system, the following equations could be obtained from (1):

$$
\begin{aligned}
& {\left[\begin{array}{llll}
m & & \\
& m & \\
& & m
\end{array}\right]\left[\begin{array}{l}
\ddot{x} \\
\ddot{y} \\
\ddot{z}
\end{array}\right]+\left[\begin{array}{lll}
C_{x} & & \\
& C_{y} & \\
& & C_{z}
\end{array}\right]\left[\begin{array}{l}
\dot{x} \\
\dot{y} \\
\dot{z}
\end{array}\right]+} \\
& {\left[\begin{array}{llll}
K_{x} & & \\
& K_{y} & \\
& & & K_{z}
\end{array}\right]\left[\begin{array}{l}
x \\
y \\
z
\end{array}\right]=\left[\begin{array}{l}
F_{x} \\
F_{y} \\
F_{z}
\end{array}\right] .}
\end{aligned}
$$

From (6), it can be seen that the translational DOFs of the system are uncoupled naturally. Thus, we only focus on the coupling situation among the translational and rotational DOFs, and the coupling situation among rotational DOFs.

If the vibration isolation system is totally uncoupled, the admittance matrix of the system must be a diagonal matrix. Correspondingly, the impedance matrix must also be a diagonal matrix.

Based on the above analysis, it can be derived that the vibration isolation system is a completely uncoupled system when the following conditions are satisfied:

$$
\begin{aligned}
& R_{x y}=R_{y z}=R_{z x}=R_{x z}=R_{z y}=R_{y x}=0 \\
& Q_{x y}=Q_{y z}=Q_{z x}=Q_{x z}=Q_{z y}=Q_{y x}=0 \\
& K_{x y}=K_{y z}=K_{z x}=K_{x z}=K_{z y}=K_{y x}=0 \\
& C_{x y}=C_{y z}=C_{z x}=C_{x z}=C_{z y}=C_{y x}=0 .
\end{aligned}
$$

The admittance matrix of the completely uncoupled system can then be obtained as

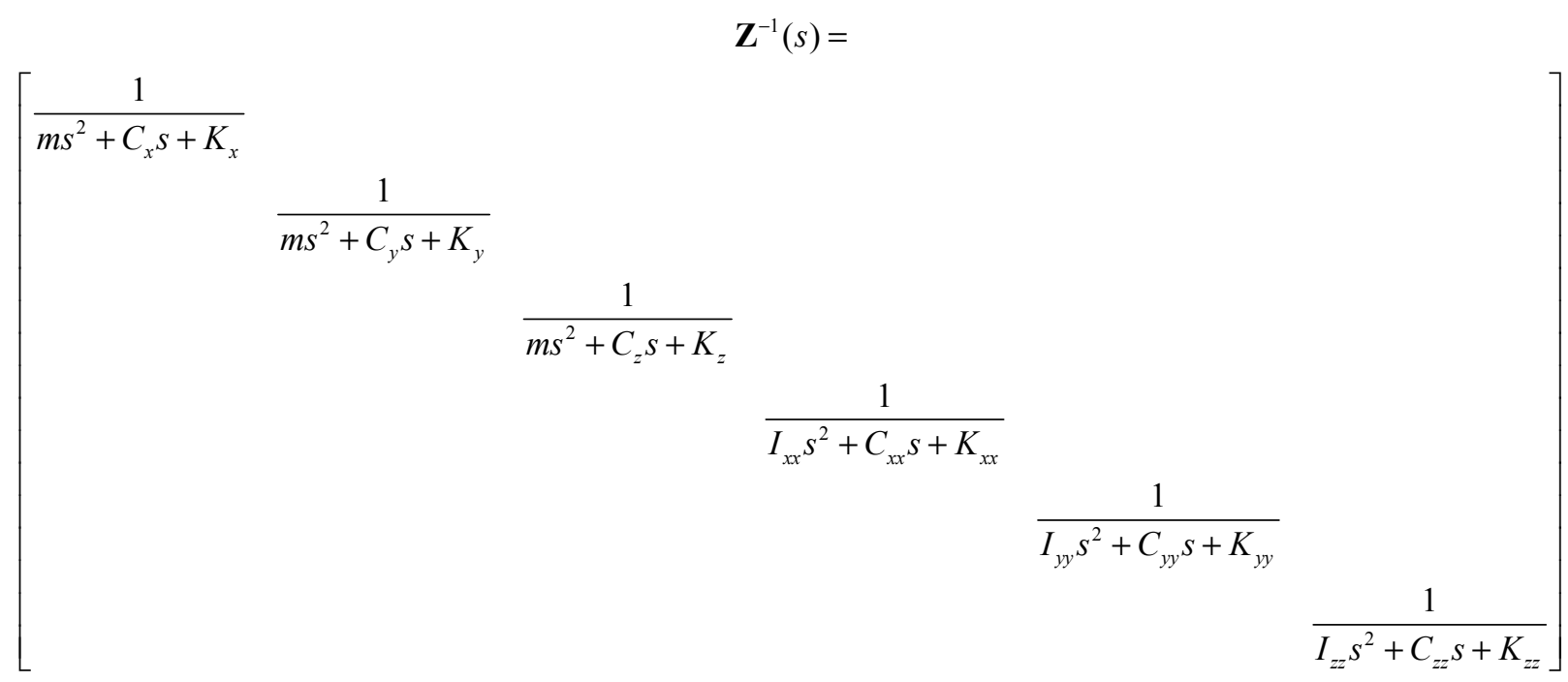

When (7) and (8) hold true and (9) and (10) do not hold true, the impedance matrix of the system can be written as (12). From (12), it can be observed that only the rotational DOFs are coupled, but the translational and rotational DOFs are uncoupled.
When (7) and (8) do not hold true and (9) and (10) hold true, the impedance matrix of the system can be written as (13). From (13), it can be observed that the rotational DOFs are uncoupled, but the translational and rotational DOFs are mutually coupled. 


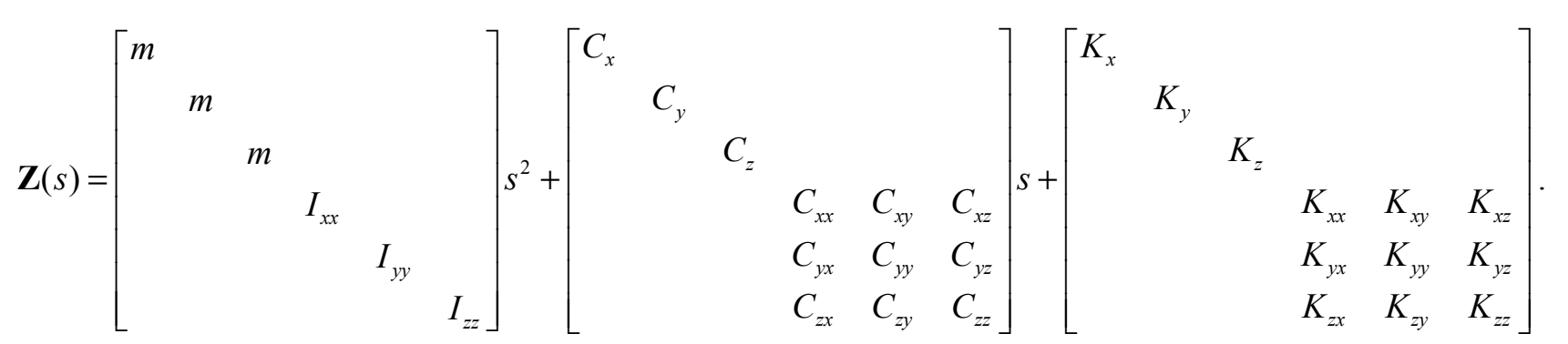

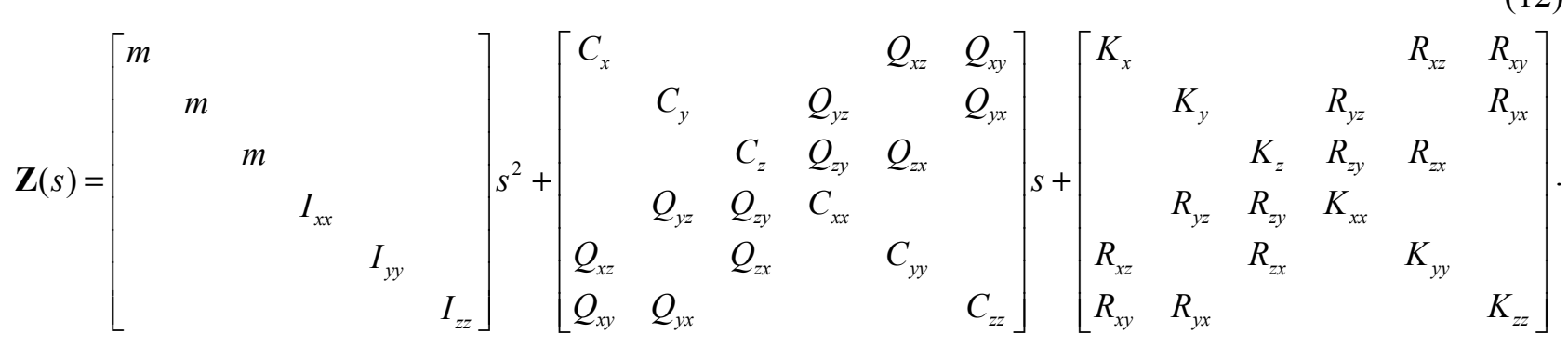

When (7) and (9) hold true and (8) and (10) do not hold true, the impedance matrix of the system can be written as (14). From (14), it can be observed that all DOFs of the stiffness matrix are uncoupled, but the translational and rotational DOFs of the damping matrix are mutually uncoupled.

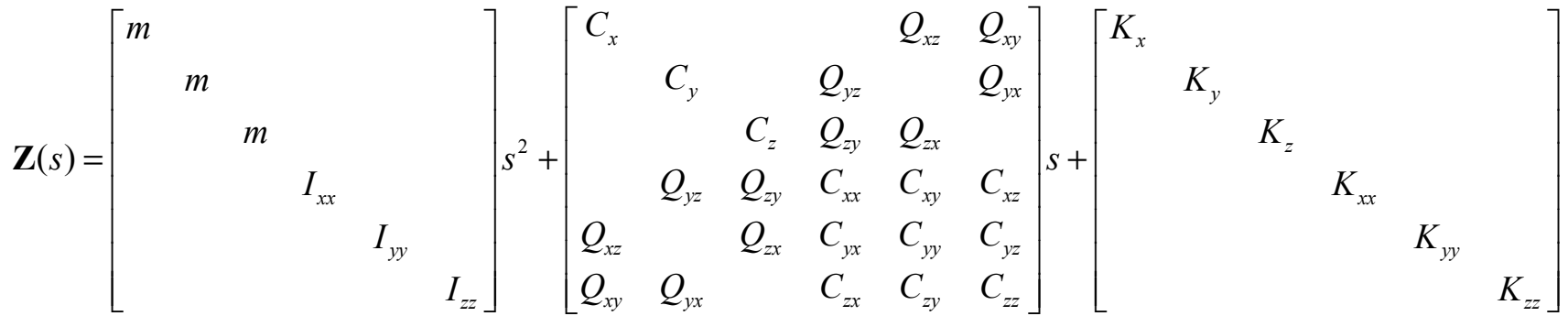

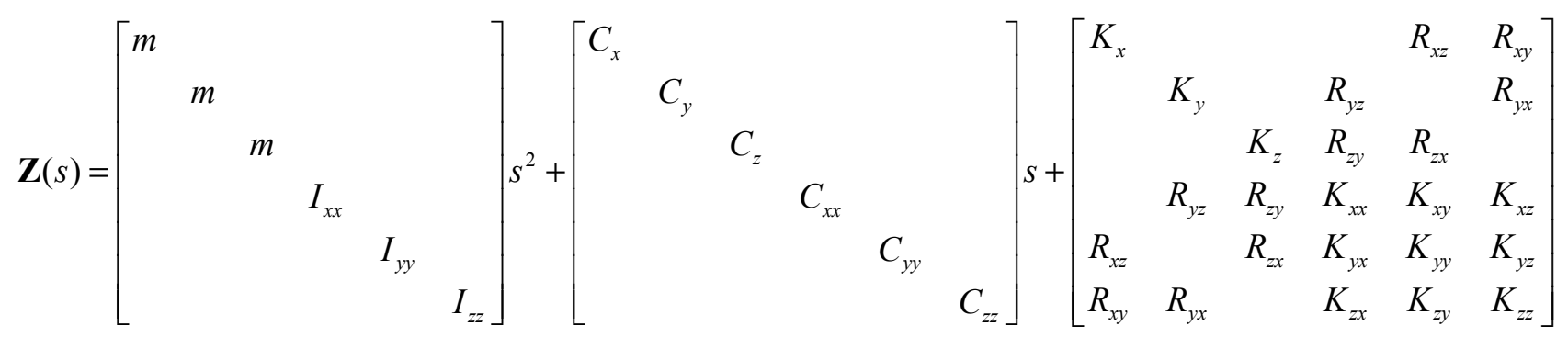

Based on the above analyses, it can be concluded that (7) to (10) formulate the criteria of determining whether the system is coupled, uncoupled, or partially coupled.

When (8) and (10) hold true and (7) and (9) do not hold true, the impedance matrix of the system can be written as (15). From (15), it can be observed that all DOFs of the damping matrix are uncoupled, but the translational and rotational DOFs of the stiffness matrix are mutually uncoupled.

. 
all the excitation loads come from the mounting The excitation load vector $\mathbf{F}$ can then be written base of the isolators in the form of motion. as

$$
\left[\begin{array}{c}
F_{x} \\
F_{y} \\
F_{z} \\
M_{x} \\
M_{y} \\
M_{z}
\end{array}\right]=\left[\begin{array}{c}
\sum_{1}^{n} c_{x i} \dot{u}_{x i} \\
\sum_{1}^{n} c_{y i} \dot{u}_{y i} \\
\sum_{1}^{n} c_{z i} \dot{u}_{i} \\
\sum_{1}^{n}\left(c_{y i} l_{z i} \dot{u}_{y i}+c_{z i} l_{y i} \dot{u}_{z i}\right) \\
\sum_{1}^{n}\left(c_{x i} l_{z i} \dot{u}_{x i}+c_{z i} l_{x i} \dot{u}_{z i}\right) \\
\sum_{1}^{n}\left(c_{x i} l_{y i} \dot{u}_{x i}+c_{y i} l_{x i} \dot{u}_{y i}\right)
\end{array}\right]+\left[\begin{array}{c}
\sum_{1}^{n} k_{x i} u_{x i} \\
\sum_{1}^{n} k_{y i} u_{y i} \\
\sum_{1}^{n} k_{z i} u_{z i} \\
\sum_{1}^{n}\left(k_{y i} l_{z i} u_{y i}+k_{z i} l_{y i} u_{z i}\right) \\
\sum_{1}^{n}\left(k_{x i} l_{z i} u_{x i}+k_{z i} l_{x i} u_{z i}\right) \\
\sum_{1}^{n}\left(k_{x i} l_{y i} u_{x i}+k_{y i} l_{x i} u_{y i}\right)
\end{array}\right]
$$

where $u_{i}$ is the excitation owing to the motion from the mounting base of Isolator $i, u_{x i}, u_{y i}$, and $u_{z i}$, are the components of $u_{i}$ along the $X-, Y$-, and $Z$-axes, respectively, as shown in Fig. 5.

From Fig. $5, u_{x i}, u_{y i}$, and $u_{z i}$ can be expressed as

$$
u_{x i}=u_{i} \cos \alpha_{i}, u_{y i}=u_{i} \cos \beta_{i}, u_{z i}=u_{i} \cos \gamma_{i} \text {. }
$$

Let $r_{x i}, r_{y i}$, and $r_{z i}$, represent $\cos \alpha_{i}, \cos \beta_{i}$, and $\cos \gamma_{i}$, respectively. From (16) and (17), the excitation load vector $\mathbf{F}$ can be expressed as (18).

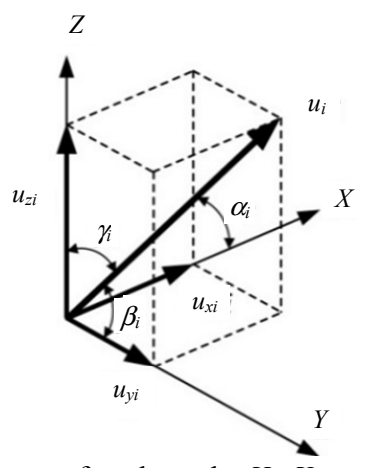

Fig. 5 Components of $u_{i}$ along the $X$-, $Y$-, and $Z$-axes.

$$
\begin{aligned}
& {\left[\begin{array}{c}
F_{x} \\
F_{y} \\
F_{z} \\
M_{x} \\
M_{y} \\
M_{z}
\end{array}\right] } {\left[\begin{array}{ccccc}
r_{x 1} c_{x 1} & \cdots & r_{x i} c_{x i} & \cdots & r_{x n} c_{x n} \\
r_{y 1} c_{y 1} & \cdots & r_{y i} c_{y i} & \cdots & r_{y n} c_{y n} \\
r_{z 1} c_{z 1} & \cdots & r_{z i} c_{z i} & \cdots & r_{z n} c_{z n} \\
r_{y 1} c_{y 1} l_{z 1}+r_{z 1} c_{z 1} l_{y 1} & \cdots & r_{y i} c_{y i} l_{z i}+r_{z i} c_{z i} l_{y i} & \cdots & r_{y n} c_{y n} l_{z n}+r_{z n} c_{z n} l_{y n} \\
r_{x 1} c_{x 1} l_{z 1}+r_{z 1} c_{z 1} l_{x 1} & \cdots & r_{x i} c_{x i} l_{z i}+r_{z i} c_{z i} l_{x i} & \cdots & r_{x n} c_{x n} l_{z n}+r_{z n} c_{z n} l_{x n} \\
r_{x 1} c_{x 1} l_{y 1}+r_{y 1} c_{y 1} l_{x 1} & \cdots & r_{x i} c_{x i} l_{y i}+r_{y i} c_{y i} l_{x i} & \cdots & r_{x n} c_{x n} l_{y n}+r_{y n} c_{y n} l_{x n}
\end{array}\right]\left[\begin{array}{c}
\dot{u}_{1} \\
\dot{u}_{2} \\
\vdots \\
\dot{u}_{i} \\
\vdots \\
\dot{u}_{n}
\end{array}\right] } \\
&+\left[\begin{array}{ccccc}
r_{x 1} k_{x 1} & \cdots & r_{x i} k_{x i} & \cdots & r_{x n} k_{x n} \\
r_{y 1} k_{y 1} & \cdots & r_{y i} k_{y i} & \cdots & r_{y n} k_{y n} \\
r_{z 1} k_{z 1} & \cdots & r_{z i} k_{z i} & \cdots & r_{z n} k_{z n} \\
r_{y 1} k_{y 1} l_{z 1}+r_{z 1} k_{z 1} l_{y 1} & \cdots & r_{y i} k_{y i} l_{z i}+r_{z i} k_{z i i} l_{y i} & \cdots & r_{y n} k_{y n} l_{z n}+r_{z n} k_{z n} l_{y n} \\
r_{x 1} k_{x 1} l_{z 1}+r_{z 1} k_{z 1} l_{x 1} & \cdots & r_{x i} k_{x i} l_{z i}+r_{z i} k_{z i i} l_{x i} & \cdots & r_{x n} k_{x n} l_{z n}+r_{z n} k_{z n} l_{x n} \\
r_{x 1} k_{x 1} l_{y 1}+r_{y 1} k_{y 1} l_{x 1} & \cdots & r_{x i} k_{x i} l_{y i}+r_{y i} k_{y i} l_{x i} & \cdots & r_{x n} k_{x n} l_{y n}+r_{y n} k_{y n} l_{x n}
\end{array}\right]\left[\begin{array}{c}
u_{1} \\
u_{2} \\
\vdots \\
u_{i} \\
\vdots \\
u_{n}
\end{array}\right]
\end{aligned}
$$

Equation(18) can thus be written as

$$
\mathbf{F}=\mathbf{A} \dot{\mathbf{U}}+\mathbf{B} \mathbf{U} \text {. }
$$

After the application of the Laplace transformation, (19) can be expressed as 


$$
\boldsymbol{\Phi}=(\mathbf{A} s+\mathbf{B}) \mathbf{U}(s)
$$

From (5) and (20), the transfer function matrix can be obtained:

$$
\mathbf{H}(s)=\frac{\mathbf{X}(s)}{\mathbf{U}(s)}=\mathbf{Z}^{-1}(s)(\mathbf{A} s+\mathbf{B}) .
$$

From (11), (18), (19), and (21), the transfer function matrix of the totally uncoupled system can be expressed in accordance to the following equation:

$$
\begin{gathered}
\mathbf{H}(s)= \\
\operatorname{diag}\left[H_{x}(s), H_{y}(s), H_{z}(s), H_{\varphi x}(s), H_{\varphi y}(s), H_{\varphi z}(s)\right]
\end{gathered}
$$

where

$$
\begin{aligned}
& H_{x}(s)=\frac{\sum_{1}^{n}\left(r_{x i} c_{x i} s+r_{x i} k_{x i}\right)}{m s^{2}+C_{x} s+K_{x}} \\
& H_{y}(s)=\frac{\sum_{1}^{n}\left(r_{y i} c_{y i} s+r_{y i} k_{y i}\right)}{m s^{2}+C_{y} s+K_{y}} \\
& H_{z}(s)=\frac{\sum_{1}^{n}\left(r_{z i} c_{z i} s+r_{z i} k_{z i}\right)}{m s^{2}+C_{z} s+K_{z}} \\
& H_{\varphi x}(s)=\frac{\sum_{1}^{n}\left[\left(r_{y i} c_{y i} l_{z i}+r_{z i} c_{z i} l_{y i}\right) s+\left(r_{y i} k_{y i} l_{z i}+r_{z i} k_{z i} l_{y i}\right)\right]}{I_{x x} s^{2}+C_{x x} s+K_{x x}} \\
& H_{\varphi y}(s)=\frac{\sum_{1}^{n}\left[\left(r_{x i} c_{x i} l_{z i}+r_{z i} c_{z i} l_{x i}\right) s+\left(r_{x i} k_{x i} l_{z i}+r_{z i} k_{z i} l_{x i}\right)\right]}{I_{y y} s^{2}+C_{y y} s+K_{y y}} \\
& H_{\varphi z}(s)=\frac{\sum_{1}^{n}\left[\left(r_{x i} c_{x i} l_{y i}+r_{y i} c_{y i} l_{x i}\right) s+\left(r_{x i} k_{x i} l_{y i}+r_{y i} k_{y i} l_{x i}\right)\right]}{s+K_{z z}} .
\end{aligned}
$$

Equation (22) is a complex function matrix. If $s$ is replaced in (22) by $\mathrm{i} \omega$, then (23) can be written as

$$
\begin{aligned}
& H_{x}(\omega)=U_{x}(\omega)+\mathrm{i} V_{x}(\omega) \\
& H_{y}(\omega)=U_{y}(\omega)+\mathrm{i} V_{y}(\omega) \\
& H_{z}(\omega)=U_{z}(\omega)+\mathrm{i} V_{z}(\omega) \\
& H_{\varphi x}(\omega)=U_{\varphi x}(\omega)+\mathrm{i} V_{\varphi x}(\omega) \\
& H_{\varphi y}(\omega)=U_{\varphi y}(\omega)+\mathrm{i} V_{\varphi y}(\omega) \\
& H_{\varphi z}(\omega)=U_{\varphi z}(\omega)+\mathrm{i} V_{\varphi z}(\omega)
\end{aligned}
$$

where $U_{x}(\omega), U_{y}(\omega), U_{z}(\omega), U_{\varphi x}(\omega), U_{\varphi y}(\omega)$, and
$U_{\varphi z}(\omega)$ represent the real components of $H_{x}(\omega)$, $H_{y}(\omega), \quad H_{z}(\omega), \quad H_{\varphi x}(\omega), \quad H_{\varphi y}(\omega), \quad$ and $H_{\varphi z}(\omega)$, respectively, and $V_{x}(\omega), V_{y}(\omega), V_{z}(\omega), V_{\varphi x}(\omega), V_{\varphi y}(\omega)$, and $V_{\varphi z}(\omega)$ represent the imaginary components of $H_{x}(\omega), H_{y}(\omega), H_{z}(\omega), H_{\varphi x}(\omega), H_{\varphi y}(\omega)$, and $H_{\varphi z}(\omega)$, respectively.

Conversion of (24) into the polar form leads to

$$
\begin{aligned}
& H_{x}(\omega)=R_{x}(\omega) \mathrm{e}^{\mathrm{i} \theta_{x}(\omega)}, H_{y}(\omega)=R_{y}(\omega) \mathrm{e}^{\mathrm{i} \theta_{y}(\omega)} \\
& H_{z}(\omega)=R_{z}(\omega) \mathrm{e}^{\mathrm{i} \theta_{z}(\omega)}, H_{\varphi x}(\omega)=R_{\varphi x}(\omega) \mathrm{e}^{\mathrm{i} \theta_{\varphi x}(\omega)} \\
& H_{\varphi y}(\omega)=R_{\varphi y}(\omega) \mathrm{e}^{\mathrm{i} \theta_{\varphi y}(\omega)}, H_{\varphi z}(\omega)=R_{\varphi z}(\omega) \mathrm{e}^{\mathrm{i} \theta_{\varphi z}(\omega)}
\end{aligned}
$$

where $R_{x}(\omega), R_{y}(\omega), R_{z}(\omega), R_{\varphi x}(\omega), R_{\varphi y}(\omega)$, and $R_{\varphi z}(\omega)$ represent the amplitudes of $H_{x}(\omega), H_{y}(\omega), H_{z}(\omega)$, $H_{\varphi x}(\omega), H_{\varphi y}(\omega)$, and $H_{\varphi z}(\omega)$, respectively; $\theta_{x}(\omega)$, $\theta_{y}(\omega), \theta_{z}(\omega), \theta_{\varphi x}(\omega), \theta_{\varphi y}(\omega)$, and $\theta_{\varphi z}(\omega)$ represent the phases of $H_{x}(\omega), H_{y}(\omega), H_{z}(\omega), H_{\varphi x}(\omega), H_{\varphi y}(\omega)$, and $H_{\varphi z}(\omega)$, respectively.

The amplitudes and phases of $H_{x}(\omega), H_{y}(\omega)$, $H_{z}(\omega), H_{\varphi x}(\omega), H_{\varphi y}(\omega)$, and $H_{\varphi z}(\omega)$ can be obtained as follows:

$$
\begin{aligned}
& R_{x}(\omega)=\sqrt{U_{x}^{2}(\omega)+V_{x}^{2}(\omega)}, \quad \theta_{x}(\omega)=\arctan \left(\frac{V_{x}(\omega)}{U_{x}(\omega)}\right) \\
& R_{y}(\omega)=\sqrt{U_{y}^{2}(\omega)+V_{y}^{2}(\omega)}, \quad \theta_{y}(\omega)=\arctan \left(\frac{V_{y}(\omega)}{U_{y}(\omega)}\right) \\
& R_{z}(\omega)=\sqrt{U_{z}^{2}(\omega)+V_{z}^{2}(\omega)}, \quad \theta_{z}(\omega)=\arctan \left(\frac{V_{z}(\omega)}{U_{z}(\omega)}\right) \\
& R_{\varphi x}(\omega)=\sqrt{U_{\varphi x}^{2}(\omega)+V_{\varphi x}^{2}(\omega)}, \theta_{\varphi x}(\omega)=\arctan \left(\frac{V_{\varphi x}(\omega)}{U_{\varphi x}(\omega)}\right) \\
& R_{\varphi y}(\omega)=\sqrt{U_{\varphi y}^{2}(\omega)+V_{\varphi y}^{2}(\omega)}, \theta_{\varphi y}(\omega)=\arctan \left(\frac{V_{\varphi y}(\omega)}{U_{\varphi y}(\omega)}\right) \\
& R_{\varphi z}(\omega)=\sqrt{U_{\varphi z}^{2}(\omega)+V_{\varphi z}^{2}(\omega)}, \theta_{\varphi z}(\omega)=\arctan \left(\frac{V_{\varphi z}(\omega)}{U_{\varphi z}(\omega)}\right)
\end{aligned}
$$

Normally, we are only concerned about the amplitude of the transfer function, which is also known as the transmissibility of the system. Transmissibility is one of the most important indicators of the vibration isolation system since it determines its performance. Therefore, it is a 
primary factor which should be considered when designing the system.

Figure 6 shows a 1-DOF passive vibration isolation system. The transmissibility of this system can be expressed as

$$
R(\omega)=\sqrt{\frac{1+4 \zeta^{2} g^{2}}{\left(1-g^{2}\right)^{2}+4 \zeta^{2} g^{2}}}
$$

where $\zeta$ is the damping ratio of the system, and $g$ is the frequency ratio. The damping ratio, the frequency ratio, and the natural frequency can be obtained in accordance to the following equations:

$$
\zeta=\frac{c}{2 m_{1-D} \omega_{n}}, \quad g=\frac{\omega}{\omega_{n}}, \quad \omega_{n}=\sqrt{\frac{k}{m_{1-D}}}
$$

where $m_{1-D}, k$, and $\omega_{n}$ are the mass, stiffness, and natural frequency of the 1-DOF passive vibration isolation system, respectively.

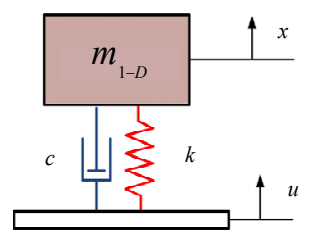

Fig. 6 Passive vibration isolation system with 1-DOF.

The curves of the transmissibility of the 1-DOF passive vibration isolation system at different damping ratios are shown in Fig. 7.

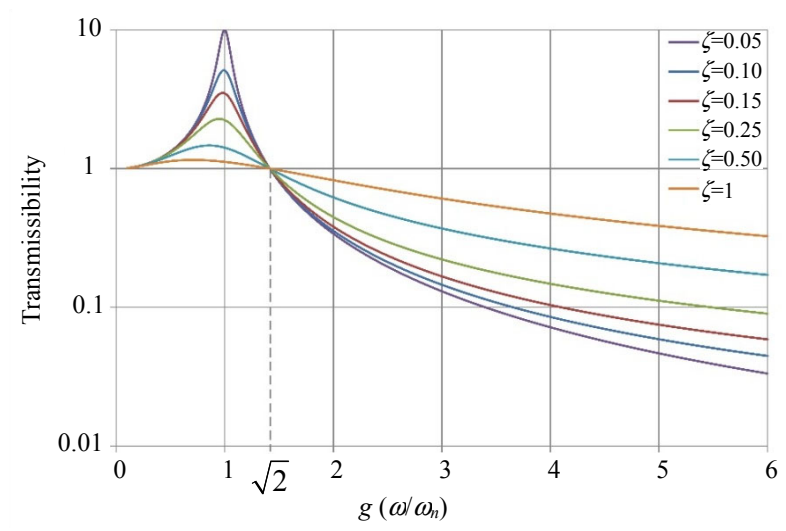

Fig. 7 Transmissibility of a 1-DOF passive vibration isolation system at different damping ratios.

From Fig. 7, it can be observed that the shape of the curve varies as a function of the damping ratio. When $g \leq \sqrt{2}$, instead of suppressing the external vibration, the system amplifies the vibration.
Moreover, the amplitude of this vibration decreases as a function of the damping ratio. When $g>\sqrt{2}$, the isolation capability of the system decreases as a function of the damping ratio. That is to say, the damping ratio and the natural frequency of the system determine the isolation performance of the system. Thus, if we set the requirement of the transmissibility, the range of the damping ratio values and the natural frequencies will be limited.

Figure 8 shows the distribution of the transmissibility with respect to the damping and frequency ratios. The blue part of the surface indicates that the transmissibility of the region is lower than $10 \%$. In other words, to make the transmissibility less than $10 \%$, the values of the damping and the frequency ratios must be within the range covered by the blue part. Furthermore, this range can be obtained from (27) in accordance with

$$
R_{r}(\omega) \geq \sqrt{\frac{1+4 \zeta^{2} g^{2}}{\left(1-g^{2}\right)^{2}+4 \zeta^{2} g^{2}}}
$$

where $R_{r}(\omega)$ is the required transmissibility.

Generally speaking, the requirement of the vibration isolation has a frequency range, e.g., the transmissibility of the isolation system is lower than $10 \%$ at frequencies of $10 \mathrm{~Hz}$ and above. Moreover, in the effective isolation range $(g)$ of the passive isolation system, the transmissibility decreases as a function of the frequency of the external vibration. Therefore, we only consider the transmissibility of the lower limit of the frequency range. Equation(29) can then be written as

$$
R_{r}(\omega) \geq \sqrt{\frac{1+4 \zeta^{2}\left(\omega_{L} / \omega_{n}\right)^{2}}{\left(1-\left(\omega_{L} / \omega_{n}\right)^{2}+4 \zeta^{2}\left(\omega_{L} / \omega_{n}\right)^{2}\right.}}
$$

where $\omega_{L}$ represents the lower limit of the frequency range.

The range of the damping ratio and the natural frequency of the required isolation system can be obtained from (30). Based on the characteristics of the isolator, an appropriate set of damping ratios and natural frequencies can be selected. 


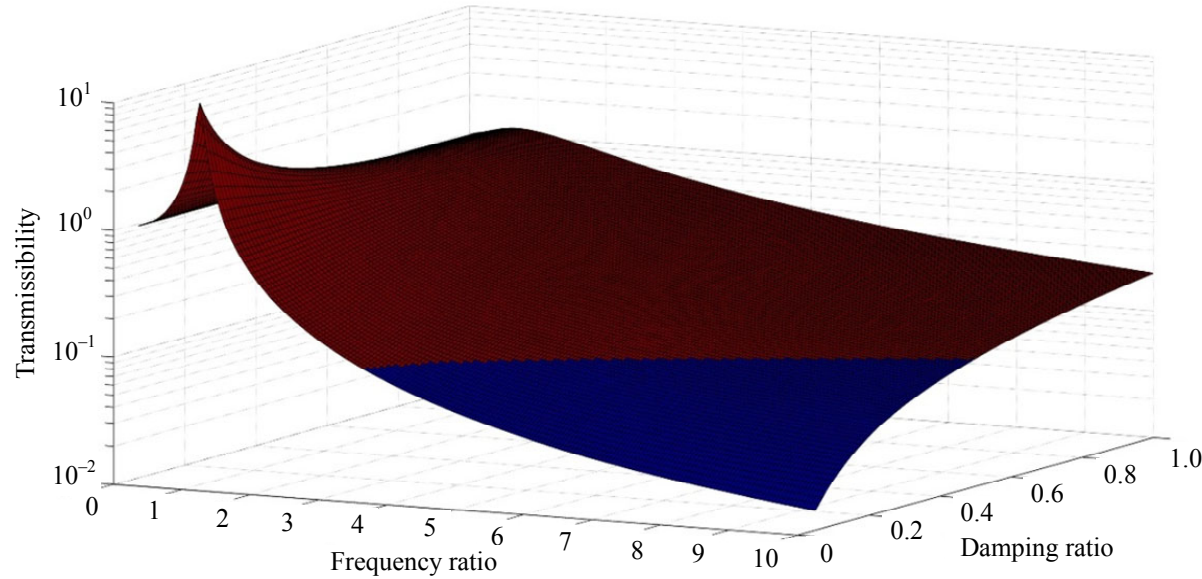

Fig. 8 Distribution of transmissibility with respect to the damping and frequency ratios.

Because the mass of the system is generally known, the stiffness and the damping coefficient of the system can be obtained from (28):

$$
k=m_{1-\mathrm{D}} \omega_{n s}^{2}, \quad c=2 m_{1-\mathrm{D}} \omega_{n s} \zeta_{s}
$$

where $\omega_{n s}$ and $\zeta_{s}$ are the selected natural frequency and damping ratio, respectively.

For an uncoupled 6-DOF isolation system, the stiffness and the damping coefficient on each DOF can be obtained from the transmissibility limits of each DOF as follows:

$$
\begin{array}{ll}
K_{x}=m \omega_{n_{-} x}^{2} ; & C_{x}=2 m \omega_{n_{-} x} \zeta_{x} \\
K_{y}=m \omega_{n_{-} y}^{2} ; & C_{y}=2 m \omega_{n_{-} y} \zeta_{y} \\
K_{z}=m \omega_{n_{-} z}^{2} ; & C_{z}=2 m \omega_{n_{-} z} \zeta_{z} \\
K_{x x}=I_{x x} \omega_{n_{-} \varphi x}^{2} ; & C_{x x}=2 I_{x x} \omega_{n_{-} \varphi x} \zeta_{\varphi x} \\
K_{y y}=I_{y y} \omega_{n_{-} \varphi y}^{2} ; & C_{y y}=2 I_{y y} \omega_{n_{-} \varphi y} \zeta_{\varphi y} \\
K_{z z}=I_{z z} \omega_{n_{-} \varphi z}^{2} ; & C_{z z}=2 I_{z z} \omega_{n_{-} \varphi z} \zeta_{\varphi z}
\end{array}
$$

where $\omega_{n_{-} x}, \omega_{n_{-} y}, \omega_{n z}, \omega_{n_{-} \varphi x}, \omega_{n_{-} \varphi y}$, and $\omega_{n_{-} \varphi z}$ are the selected natural frequencies of each DOF, respectively, and $\zeta_{x}, \zeta_{y}, \zeta_{z}, \zeta_{\varphi x}, \zeta_{\varphi y}$, and $\zeta_{\varphi z}$ are the selected damping ratios of each DOF, respectively.

The designer of the vibration isolation system could choose to limit the transmissibility of all or some DOFs, based on the different application requirements. The constraint equations of the design will then be obtained.

\section{Layout constraints}

In addition to the above considerations, the number and layout of the isolators of a vibration isolation system must be restricted by the size and shape of the payload and the installation space. For example, a triangularly shaped payload is usually supported by three isolators on three corners, as shown in Fig. 9(a). For a payload with asymmetrical appearance, the isolators are usually symmetrically arranged, as shown in Fig. 9(b). Correspondingly, a circularly shaped payload is usually supported by three or more evenly arranged isolators, as shown in Fig. 9(c).

The examples described above are only general cases, and designers can make appropriate changes based on the actual situations.

Figure 10(a) shows a payload supported by two isolators. From Fig. 10(a), the distance between Isolators 1 and 2 is changeable, and the range of the distances can be expressed as follows:

$$
L_{\min } \leq l_{1}+l_{2}=L \leq L_{\max }
$$

where $O$ is the center of mass of the payload; $m_{1}$ is the mass of the payload; $L$ is the installation distance between Isolators 1 and $2 ; l_{1}$ and $l_{2}$ are the distances from Isolator 1 and Isolator 2 to the center of mass, respectively; $L_{\min }$ and $L_{\max }$ are the minimum and maximum installation distance between Isolators 1 and 2 , respectively. 


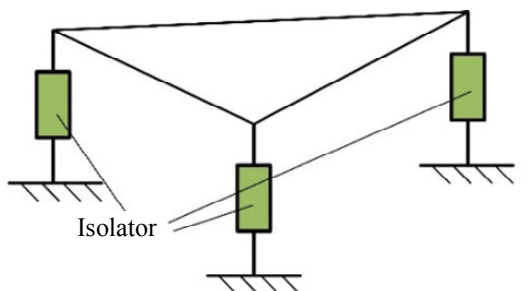

(a)

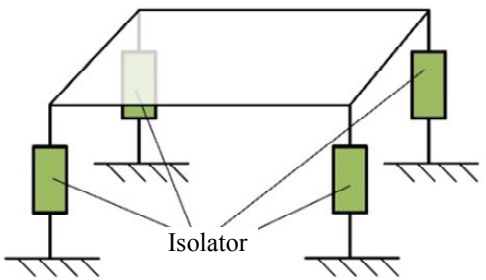

(b)

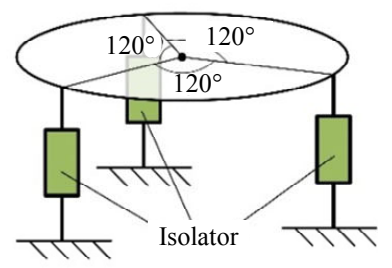

(c)

Fig. 9 Several different types of isolators layout: (a) triangularly shaped payload supported by three isolators, (b) rectangular payload supported by four symmetrically arranged isolators, and (c) circularly shaped payload supported by three evenly arranged isolators.

(a)

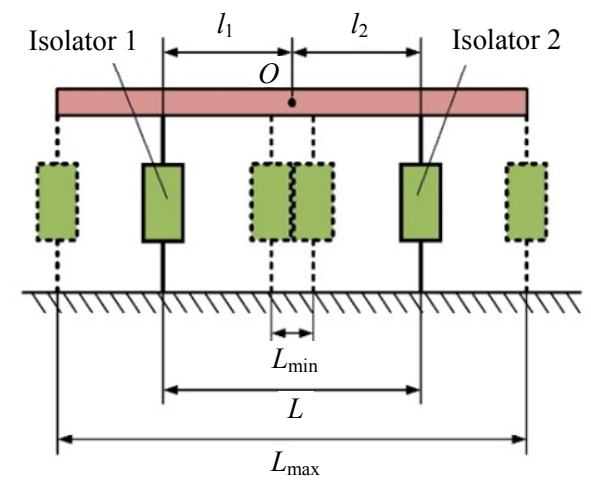

(b)

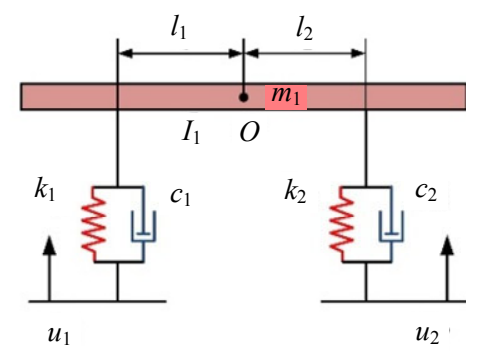

Fig. 10 2-DOF passive vibration isolation system: (a) a payload supported by two isolators and (b) schematic diagram of the 2-DOF passive vibration isolation system.

This system can be treated as a 2-DOF passive vibration isolation system, as shown Fig. 10(b). If we consider that this system is an uncoupled system, the following equations can be obtained:

$$
c_{1} l_{1}=c_{2} l_{2}, \quad k_{1} l_{1}=k_{2} l_{2}
$$

where $m_{1}$ is the mass of the 2-DOF passive vibration isolation system; $c_{1}$ and $c_{2}$ are the damping coefficients of Isolators 1 and 2, respectively; $k_{1}$ and $k_{2}$ are the stiffnesses of Isolators 1 and 2 , respectively.

The dynamic equations of this 2-DOF passive vibration isolation system can be obtained as

$$
\left[\begin{array}{ll}
m_{1} & \\
& I_{1}
\end{array}\right]\left[\begin{array}{l}
\ddot{x} \\
\ddot{\theta}
\end{array}\right]+\left[\begin{array}{ll}
c_{1}+c_{2} & \\
& c_{1} l_{1}^{2}+c_{2} l_{2}^{2}
\end{array}\right]\left[\begin{array}{l}
\dot{x} \\
\dot{\theta}
\end{array}\right]+\left[\begin{array}{ll}
k_{1}+k_{2} & \\
& k_{1} l_{1}^{2}+k_{2} l_{2}^{2}
\end{array}\right]\left[\begin{array}{l}
x \\
\theta
\end{array}\right]=\left[\begin{array}{c}
\left(c_{1} \dot{u}_{1}+c_{2} \dot{u}_{2}\right)+\left(k_{1} u_{1}+k_{2} u_{2}\right) \\
\left(c_{1} l_{1} \dot{u}_{1}+c_{2} l_{2} \dot{u}_{2}\right)+\left(k_{1} l_{1} u_{1}+k_{2} l_{2} u_{2}\right)
\end{array}\right] \text {. }
$$

If $a=c_{1} l_{1}=c_{2} l_{2}$ and $b=k_{1} l_{1}=k_{2} l_{2},(35)$ can be written as

$$
\left[\begin{array}{ll}
m_{1} & \\
& I_{1}
\end{array}\right]\left[\begin{array}{c}
\ddot{x} \\
\ddot{\theta}
\end{array}\right]+\left[\begin{array}{cc}
c_{1}+c_{2} & \\
& a\left(l_{1}+l_{2}\right)
\end{array}\right]\left[\begin{array}{c}
\dot{x} \\
\dot{\theta}
\end{array}\right]+\left[\begin{array}{ll}
k_{1}+k_{2} & \\
& b\left(l_{1}+l_{2}\right)
\end{array}\right]\left[\begin{array}{c}
x \\
\theta
\end{array}\right]=\left[\begin{array}{c}
\left(c_{1} \dot{u}_{1}+c_{2} \dot{u}_{2}\right)+\left(k_{1} u_{1}+k_{2} u_{2}\right) \\
a\left(\dot{u}_{1}+\dot{u}_{2}\right)+b\left(u_{1}+u_{2}\right)
\end{array}\right] .
$$

The transmissibility of the rotational DOF can be derived from (23) to (26):

$$
R_{\theta}(\omega)=\sqrt{\frac{4 a^{2} \omega^{2}+4 b^{2}}{\left[b\left(l_{1}+l_{2}\right)-I_{1} \omega^{2}\right]^{2}+a^{2} \omega^{2}\left(l_{1}+l_{2}\right)^{2}}} .
$$

From (33) and (34), $a$ and $b$ can be expressed as

$$
a=\frac{c_{1} c_{2}}{c_{1}+c_{2}} L, \quad b=\frac{k_{1} k_{2}}{k_{1}+k_{2}} L
$$

Substituting (8) into (37), the transmissibility of 
the rotational DOF can be expressed as

$$
\begin{aligned}
R_{\theta}(\omega) & =\sqrt{\frac{4 \omega^{2}+4\left(\frac{k_{1}}{c_{1}}\right)^{2}}{\left(\frac{k_{1}}{c_{1}} L-\frac{\left(c_{1}+c_{2}\right) I_{1}}{c_{1} c_{2} L} \omega^{2}\right)^{2}+\omega^{2} L^{2}}} \\
= & \sqrt{\frac{4\left(\frac{c_{1}}{k_{1}}\right)^{2} \omega^{2}+4}{\left(L-\frac{\left(k_{1}+k_{2}\right) I_{1}}{k_{1} k_{2} L} \omega^{2}\right)^{2}+\left(\frac{c_{1}}{k_{1}}\right)^{2} \omega^{2} L^{2}}} .
\end{aligned}
$$

From (39), it can be seen that the transmissibility of the rotational vibration is influenced by the distance between isolators. The larger the distance is, the better the isolation effect of the rotational vibration is. Therefore, it is important to try to make full use of the installation space when designing a vibration isolation system.

\section{Conclusions}

The performance of an optical system with a sensitive LOS is heavily influenced by the rotational vibration. Thus, the primary task of designing the vibration isolation systems for these optical systems is to reduce as much as possible or eliminate the rotational vibration. The design methodology of a passive vibration isolation system for an optical system with the sensitive LOS is proposed herein. The main steps of the design process are as follows:

(1) The design of a vibration isolation system must be restricted by the size and shape of the payload and the installation space. The number and layout of the isolators of a vibration isolation system should be firstly determined based on these restrictions. It is important to make full use of the installation space. This step is referred to as "layout constraints".

(2) To reduce the effect of the rotational vibration, the rotational vibration caused by the coupling is reduced or eliminated first. Equations(7) to (10) represent the decoupling conditions of the system. The coupling vibration is eliminated when the parameters of the isolators fulfill (7) to (10). This step is referred to as "decoupling constraints".

(3) Transmissibility is one of the most important indicators of the vibration isolation system since it determines the performance of the system. When the system is totally uncoupled, the transmissibility on each DOF is only determined by the stiffness and damping coefficients of the system on the corresponding DOF. Thus, the stiffness and damping coefficient on each DOF of the system can be obtained by limiting the transmissibility on each DOF based on the actual design requirements. This step is known as "transmissibility constraints".

In most cases, a unique solution cannot be obtained, even if all three types of constraints are met. However, it offers designers the necessary designing freedom to overcome other unexpected constraints.

\section{Acknowledgment}

This work was supported by the Strategic Priority Research Program of the Chinese Academy of Science (Grant No. XDA1502070404) and the Projects of Science Technology Development Plan of Jilin Province (Grant No. 20190302102GX).

Open Access This article is distributed under the terms of the Creative Commons Attribution 4.0 International License (http://creativecommons.org/licenses/by/4.0/), which permits unrestricted use, distribution, and reproduction in any medium, provided you give appropriate credit to the original author(s) and the source, provide a link to the Creative Commons license, and indicate if changes were made.

\section{References}

[1] J. Yang, Z. Xu, Q. Wu, M. Zhu, S. He, and C. Qin, "Dynamic modeling and control of a 6-DOF micro-vibration simulator," Mechanism and Machine Theory, 2016, 104: 350-369.

[2] X. Sun, B. Yang, L. Zhao, and X. Sun, "Optimal design and experimental analyses of a new micro-vibration control payload-platform," Journal of Sound and Vibration, 2016, 374: 43-60.

[3] B. Lam and C. Guo, "Complete characterization of 
ultrashort optical pulses with a phase-shifting wedged reversal shearing interferometer," Light: Science \& Applications, 2018, 7: 30.

[4] Y. Liu, X. Fu, L. Xu, and Y. Song, "Design and finite element analysis of the vibration isolation system of STM," SPIE, 2013, 8916: 89160Y.

[5] X. L. Yang, H. T. Wu, Y. Li, and B. Chen, "Dynamic isotropic design and decentralized active control of a six-axis vibration isolator via Stewart platform," Mechanism and Machine Theory, 2017, 117: 244-252.

[6] F. Matichard, B. Lantzc, K. Mason, R. Mittleman, B. Abbott, S. Abbott, et al., "Advanced LIGO two-stage twelve-axis vibration isolation and positioning platform. Part 1: Design and production overview," Precision Engineering, 2015, 40: 273-286.

[7] Y. Wu, K. Yu, J. Jiao, and R. Zhao, "Dynamic modeling and robust nonlinear control of a six-DOF active micro-vibration isolation manipulator with parameter uncertainties," Mechanism and Machine Theory, 2015, 92: 407-435.

[8] J. L. Miller, S. P. Way, B. Ellison, and C. L. Archer, "Design challenges regarding high-definition electro-optic/infrared stabilized imaging system," Optical Engineering, 2013, 52(6): 061310.

[9] B. Agrawal, "Jitter control for imaging spacecraft," in Proceedings of 2009 4th International Conference on Recent Advances in Space Technologies, Istanbul, 2009, pp. 615-620.

[10] Z. Y. Xue, B. Qi, and G. Ren, "Vibration-induced jitter control in satellite optical communication," SPIE, 2013, 8906: 89061X.

[11] D. Kamesh, R. Pandiyan, and A. Ghosal, "Modeling, design and analysis of low frequency platform for attenuating micro-vibration in spacecraft," Journal of Sound and Vibration, 2010, 329(17): 3431-3450.

[12] A. J. Bronowicki and J. W. Innis, "A family of full spacecraft-to-payload isolators," Technology Review Journal, 2005, 13: 21-41.

[13] Z. Wu, X. Jing, B. Sun, and F. Li, "A 6DOF passive vibration isolator using X-shape supporting structures," Journal of Sound and Vibration, 2016, 380: 90-111.

[14] A. G. Piersol and T. L. Paez, Harris shock and vibration handbook (sixth edition). New York: McGraw-Hill, 2009.

[15] C. Shi, R. G. Parker, and S. W. Shaw, "Tuning of centrifugal pendulum vibration absorbers for translational and rotational vibration reduction," Mechanism and Machine Theory, 2013, 66: 56-65.

[16] M. J. Krizo, S. J. Cusumano, S. T. Fiorino, R. Heap, V. Velten, J. Brown, et al., "Design, development, and in-flight testing of a pointer/tracker for in-flight experiments to measure aero-optical effects over a scaled turret," Optical Engineering, 2013, 52(7): 071415.

[17] K. B. Doyle, "Structural line-of-sight jitter analysis for MLCD," SPIE, 2007, 6665: 66650I.

[18] D. O. Lee, J. S. Yoon, and J. H. Han, "Integrated framework for jitter analysis combining disturbance, structure, vibration isolator and optical model," SPIE, 2012, 8341: 834126.

[19] X. C. Li, J. C. Hamann, and J. E. McInroy, "Simultaneous vibration isolation and pointing control of flexure jointed hexapods," SPIE, 2001, 4327: 99-109.

[20] L. Vaillon and C. Philippe, "Passive and active microvibration control for very high pointing accuracy space systems," Smart Materials and Structures, 1999, 8(6): 719-728.

[21] D. O. Lee, J. S. Yoon, and J. H. Han, "Integrated framework for jitter analysis combining disturbance, structure, vibration isolator and optical model," SPIE, 2012, 8341: 834126.

[22] C. Blaurock, K. C. Liu, L. Dewell, and J. Alexander, "Passive isolator design for jitter reduction in the terrestrial planet finder coronagraph," SPIE, 2005, 5867: 58670Y.

[23] V. Sannibale, G. G. Ortiz, and W. H. Farr, "A sub-hertz vibration isolation platform for a deep space optical communication transceiver," SPIE, 2009, 7199: 71990I.

[24] H. U. Oh, S. C. Kwon, and S. H. Youn, "Characteristics of spaceborne cooler passive vibration isolator by using a compressed shape memory alloy mesh washer," Smart Materials and Structures, 2014, 24(1): 015009.

[25] M. B. McMickell, T. Kreider, E. Hansen, T. Davis, and M. Gonzalez, "Optical payload isolation using the miniature vibration isolation system (MVIS-II)," SPIE, 2007, 6527: 652703.

[26] C. Liu, X. Jing, S. Daley, and F. Li, "Recent advances in micro-vibration isolation," Mechanical Systems and Signal Processing, 2015, 56: 55-80.

[27] P. Davis, D. Cunningham, and J. Harrell, "Advanced $1.5 \mathrm{~Hz}$ passive viscous isolation system," in Proceedings of the 35th AIAA SDM Conference Hilton Head, South Carolina, April 18-20, 1994, pp. $1-11$.

[28] R. G. Cobb, J. M. Sullivan, A. Das, L. P. Davis, T. T. Hyde, T. Davis, et al., "Vibration isolation and suppression system for precision payloads in space," Smart Materials and Structures, 1999, 8(6): 798-812.

[29] J. O'Toole, "Passive vibration isolation for long range aerial reconnaissance systems," SPIE, 1980, 250: 77-86.

[30] T. T. Hyde, "A conceptual design methodology for vibration isolation," SPIE, 1997, 3041: 760-771. 